Working Paper n. 34

Massimo Giannini

\title{
HUMAN CAPITAL AND INCOME DISTRIBUTION DYNAMICS
}

Roma, marzo 2000 


\begin{abstract}
The paper assumes a continuum of two period-lived agents; agents are identical except for inherited income. Young agents allocate their inheritance between consumption and investment in human capital under uncertainty. In the second period they receive a wage proportional to the accumulated human capital and invest in offspring. Two main results arise: a low earning per unit of human capital leads economy to converge to a stationary income distribution whatever the initial distribution. Viceversa, for a sufficiently high wage an endogenous growth operates and the distributive dynamics depends on initial conditions. In this case different redistributive policies are analyzed.
\end{abstract}

J.E.L. Classification: D31, D90, O15, O41

Keywords: human capital accumulation, personal income distribution, diffusion processes, Fokker-Planck. 


\title{
HUMAN CAPITAL AND INCOME DISTRIBUTION DYNAMICS
}

\author{
Massimo Giannini ${ }^{\circ}$
}

\section{1 - Introduction}

Aim of this paper is to develop an analytical framework investigating the dynamics of income distribution through investment in human capital, as in the spirit of Galor and Zeira, (1993), Glomm and Ravikumar, (1992), Benabou, (1996) and Galor and Tsiddon, (1997), among others. The analysis of the personal income distribution arises several questions and the paper tries to investigate some leading points related both to thereotical and empirical questions. The first one concerns the effects, on the personal income distribution, of different opportunities in accumulating human capital as a result of different initial conditions; in a "free-market", under certain assumptions, such heterogeneity causes inequality among individuals, and such inequality can be amplified over time, especially when intergenerational transfers are at work, inducing a path-dependance in the dynamics of the income curve. This kind of question brings us to the following: "Is the long run dynamics affected by initial conditions?" In the literature both a positive and a negative answer can be found: the Loury's article, (1981), showed that inequalities can affect the income curve in the

\footnotetext{
*University of Rome "Tor Vergata", Dip. Dir. Proc. Civ., Via Orazio Raimondo 18, 00173 Rome-Italy. E-mail: Massimo.Giannini@uniroma2.it. Acknowledgements. I wish to thank Claudio Gnesutta, Vincenzo Denicolò, partecipants to various seminars and at the 11th conference of the European Economic Association for helpful suggestions and comments
} 
short run but they have no influence in the long term, where the income distribution achieves an ergodic measure. Later, Galor and Zeira (1993) have shown that the joint presence of thresholds in human capital investment and of imperfect capital markets causes inequalities to affect the short as well as the long run dynamics. Recently, Benabou, (1996) showed that equality tends to slow down growth in the short run but the nexus is reversed in the long term. These results underline the importance for a complete analysis of the income curve dynamics stressing both the short and the long run properties: this is the goal of the first part of the work where convergence to an ergodic distribution can occur or not according to a parametric change on the labour market. Another relevant point about the dynamics of the income distribution is the lack of a steady trend, as the empirical observation seems to suggest;

The evidence ..... suggests that it may be better for a number of countries to think in terms of "episodes" when inequality fell or increased.....there was a widespread belief that inequality was falling, steadily ...As we know now, income inequality did not continue to fal ${ }^{2}$

According to a OECD empirical study (1993), the dynamics of the income curve in the past two decades, for a large number of countries, was characterized by an alternating fall and rise in income dispersion ${ }^{1}$. Reasons

on an earlier draft. Moreover, I am particularly indebted to Anthony Atkinson for advices, stimuli and material on the subject. Obviously, any errors are attributable solely to myself.

${ }^{2}$ A.B. Atkinson (1996) page 6.

${ }^{3}$ In a recent empirical work, Perotti, (1996), tries to shed a light on the relationship growthequality. He finds a broad positive association between the growth rate and equality, although with some caveats. The sample under investigation covers from 1960 to 1985 but the second half of ' 80 was characterized by a strong increase in inequalities indexes, especially in USA and UK. 
for this behaviour can be very complex but undoubtedly a part of the story is closely related to a change in the economical structure. To take into account such "stylized fact", a theoretical scheme should be able to provide different dynamic behaviours according to a slight change in the basic assumptions describing the postulated economic system. Two recent works achieve the goal, according to some parametric change. In the Acemoglu's work, (1997), inequality increases or decreases according to frictions on the labour market: the more efficient the labour market, the higher the inequality and lower the growth rate and viceversa. Hence according to imperfections on the labour market, measured by the length of search, it is possible to take into consideration both increasing and decreasing inequality jointly to a high or low growth. In the Galor and Tsiddon's work, (1997), instead the result comes from non-linearities in the accumulation of human capital, i.e. from the presence of multiple steady-states. This non-linearity, jointly to a particular technological progress, causes the income curve to change from a bimodal to a unimodal distribution over time, reducing inequality. Our model can generate both increasing and decreasing inequality, jointly to an endogenous growth and a stationary economy respectively, according to the human capital remuneration on a Walrasian labour market, hence without assuming imperfections in such market. Moreover the human capital accumulation technology, for each dynasty, is linear, hence characterized by a monotonic evolution. In other words, the result comes from a theoretical framework which is as simple as possible. Under these simple assumptions the paper provides a rigorous analytical study of the statistical law describing the income curve dynamics which, to the best of my knowledge, 
is missing in the literature. Since both a fall and a rise in dispersion are possible in our model according to a parametric change, the paper concludes for a trade-off between growth and equality. For such a reason the second part focuses on some examples of redistributive policies aiming to investigate the effect on the growth process of transfer actions. Despite the widespread belief that welfare policies represent an obstacle to the growth process, although there is not a clear reason supporting such a conclusion (see Atkinson, 1995) the second part of the work shows as such policies can reduce inequality without slowing down the growth rate. It must be stressed that the empirical work of Perotti concludes that ...social security expenditure is positively, rather than negatively, associated with growth...' (Perotti, 1996, page 161). The paper is articulated as follows: in section two and three both the basic model and the individual behaviour are formalized. In section 4 and 4.1 the assumption of individuals homogeneity is relaxed in order to investigate the statistical law underlying the income distribution and the relationship with the initial conditions; in section 4.2 the income distribution moments are analyzed providing, jointly with the previous point, a full characterization of the temporal properties of the income curve. Section 5 summarizes the results while section 6 analyzes the effect of different schemes of redistributive policies on the growth process.

\section{2 - The model}

The economy is populated by a continuum of two period-lived, heterogenous agents living in overlapping generations. Each parent has one child, which provides the relationship between generations within dynasties; 
individual is child when young and parent when old. These assumptions imply a stationary population whose size is normalized to one. Moreover, parents take care of offspring by transferring them their income (net of consumption); this sort of parental investment provides the endowment for the young agents (in the following we will mean this parental investment as an inheritance). In their first period of life individuals decide how to allocate their inherited income (parental investment) between consumption and investment in education (human capital); human capital is forgone consumption. Agents are identical with respect to their preferences and differ only with respect to their inherited income. Consequently, the inheritance of each individual affects the amount of human capital she accumulates. Differently from Galor and Zeira (1993), each individual invests in human capital, although in different levels. In the second half of their life agents work, receiving a wage which is proportional to the acquired human capital; this earning is allocated between consumption, which is proportional to the inherited income according to a fixed propensity, and investment in offspring. Differently from the standard models of human capital accumulation (as for example Uzawa, 1965, and Lucas, 1988), where a specific production sector for human capital exists, I assume a simple one-to-one relationship between income and human capital; a unit of inherited income "buys" a unit of human capital. The latter is the only way to transfer income from the first to the second period of life, since human capital is the only "asset" in this simple economy and borrowing is denied. These assumptions allow us to focus only on the intergenerational transfer of earnings related to human capital accumulation, 
rather than to a wealth context, which is well investigated both in Loury (1981) and Galor and Zeira (1993). Finally, human capital is homogenous and there is only one type of it; agents differ only in size but not in composition. As we said, during the second period of life, agents receive a wage proportional to the accumulated human capital; more educated people gain a higher earning (college premium). I assume that the economy is equipped with a linear production function producing the only good in such an economy and whose only argument is human capital: $Y=A h$ which represents a constant returns to scale technology on the accumulable input. In this simple economy hence each agent receives a "wage" $A h^{i}$ where $h^{i}$ is the human capital accumulated by the $i$-th agent during the youth. Moreover I assume that the wage is subject to a random shock which captures the uncertainty on the labour market or other factors related to "chance".

\section{3 - The individual behaviour}

In this section we are going to analyze the individual behaviour. As we said, each individual has to perform her human capital investment according to a maximizing program. Agents born at time $t$, maximize a utility function, whose arguments are the first period consumption $C_{t}$ and the investment in offspring $X_{t+1}$. The individual program is then:

$$
V_{t}\left(X_{t}\right)=\operatorname{Max}_{C}\left\{U_{1}\left(C_{t}\right)+\beta E\left[U_{2}\left(X_{t+1}\right) \mid t\right]\right\}
$$

subject to the following life cycle:

$$
\begin{aligned}
& C_{t}=X_{t}-h_{t} \\
& W_{t+1}=A h_{t}+\gamma \varepsilon_{t+1}
\end{aligned}
$$




$$
\begin{aligned}
& X_{t+1}=W_{t+1}-S \\
& C t, X t, h t, X t+1>0 \forall t
\end{aligned}
$$

where $C$ stands for consumption, $X$ for income, $h$ for human capital, $W$ for wage, $A$ is the mean wage for unit of human capital, $S=\theta X t, \theta<1$, is the fixed-level consumption for the second period ${ }^{4}$ and $\beta \in(0,1)$ is the second period utility weight. $\varepsilon_{t+1}$ is an instantaneous wage shock. We shall assume a stationary, gaussian i.i.d. $(0,1)$ random process; $\gamma$ is a positive coefficient measuring the local impact of the noise on the wage. Finally we assume $U^{\prime}>0, U^{\prime \prime}<0$ and $U^{\prime \prime \prime}>0$. The assumption that individuals consume in the second period according to a fixed propensity rather than to an optimal choice is not relevant, although likely less elegant. As it will be clear in the following, the dynamics depends on the non-linearity of the optimal plans w.r.t. the coefficient $A$ and such a result is robust to more complex maximizing programs which have not explicit solution in presence of the random shock. In (2) the first period income $X_{t}$ (the parental investment), for agents born in period $t$, is exhaustively allocated between consumption and investment in human capital. In (4) the bequest $X_{t+1}$ comes from the difference between wage, which is proportional to the accumulated human capital plus a random term, and the fixed-consumption $S$. The $X_{t+1}$ income provides the endowment (investment) of the offspring. Since an explicit solution is necessary in order to study the dynamics of the income curve, I

\footnotetext{
${ }^{4}$ The assumption about the relationship between second period consumption and inherited income is not obviously crucial and we can also assume that people consume the same level during the old age. Nevertheless it seems to me more realistic assuming that individuals try to mantain the "life-style" also during the second period. For this reason I assume that individuals consume in a heterogenous way in the second period, according to their "social rank".
} 
assume a constant absolute risk aversion utility function, $U(z)=-(1 / \alpha) \exp (-$ $\alpha z$ ), where $\alpha$ is the absolute risk aversion coefficient. It must be remarked anyway, that this particular form of preferences does not alter the dynamical property allowing, at the same time, an explicit solution to the maximization problem which is necessary to study the dynamics. What is important in such a context is only the concavity property of the utility function. Solving the optimization program we obtain $C_{t}{ }^{*}$, and from the (1) $h_{t}{ }^{*}$ :

$$
\begin{aligned}
C^{*}{ }_{t} & =\frac{a_{2}(A-\theta)}{a_{1}+a_{2} A} X_{t}-\frac{2 \ln (A \beta)+\left(a_{2} \gamma\right)^{2}}{2\left(a_{1}+a_{2} A\right)} \\
h^{*}{ }_{t} & =\frac{a_{1}+a_{2} \theta}{a_{1}+a_{2} A} X_{t}+\frac{2 \ln (A \beta)+\left(a_{2} \gamma\right)^{2}}{2\left(a_{1}+a_{2} A\right)}
\end{aligned}
$$

where $a_{i} i=1,2$ is the absolute risk aversion coefficient (curvature) of the sub-utility functions. Consumption is a linear function of $X_{t}$; it is worth stressing the role played by uncertainty in (5): the higher the uncertainty $(\gamma)$, the lower the current consumption, which in turn involves a higher human capital; the rationale lies in the well-known precautionary behaviour. Nevertheless the effect of uncertainty tends to disappear as income grows; richer people are less worried about future so as to prefer consumption to human capital investment. Finally we remark that the positive constraints on the variables imply a minimum inherited income, $X$, and $A>\theta$. Both (5) and (6) are linear in $X_{t}$ but non-linear w.r.t. $A$. In particular the derivative of $h^{*}$ w.r.t. $A$ changes from a positive to a negative value for some $A^{\prime}$ in $R^{+}$ because of the income and substitution effects; for $A>A^{\prime}$ the income effect prevails and in this way we obtain $d h^{*} / d A<0$, which in turn implies $d C^{*} / d A>0$. Moreover we have $d A^{\prime} / d X<0$; the richer the individual, the lower 


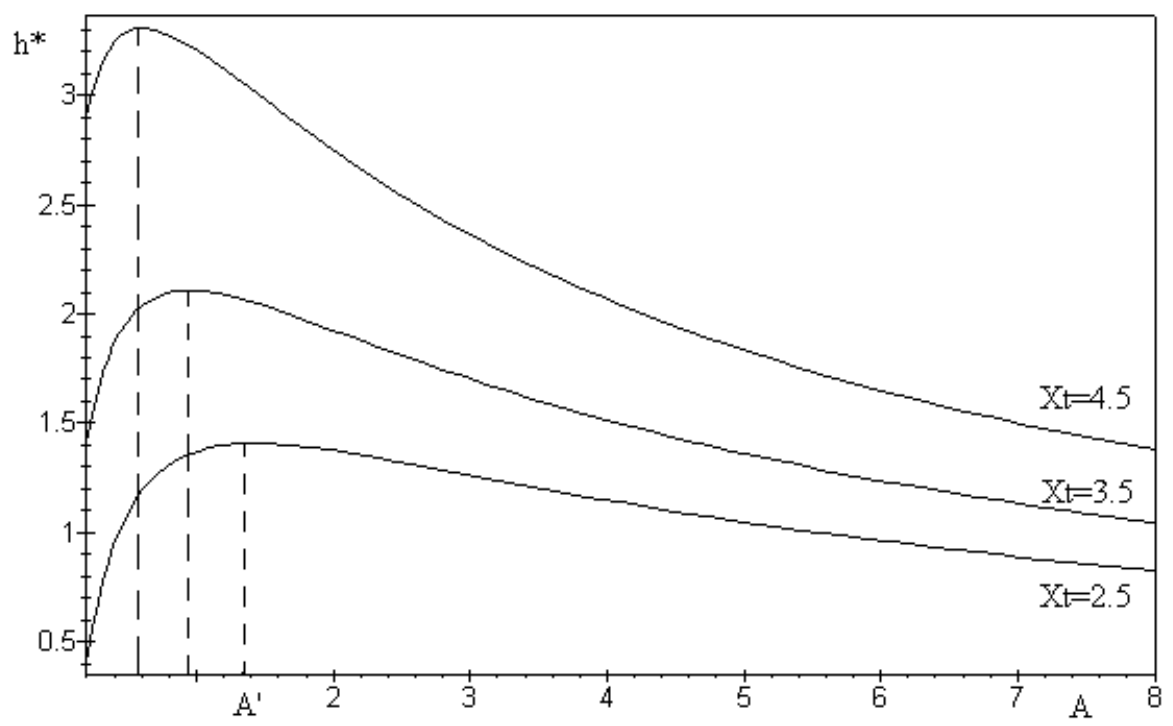

Figure 1

the subjective threshold $A^{\prime}$ (see figure 1); as noted, individuals characterized by a high inheritance prefer consumption to human capital accumulation. Hence, according to the mean wage, individuals can prefer consumption to human capital or viceversa; because of the intergenerational transfer, this behaviour affects the whole dynasty.By (3), (4) and (2) we obtain the following stochastic difference equation:

$$
X^{*}{ }_{t+1}=(A-\theta) X_{t}-A C^{*}{ }_{t}+\gamma \varepsilon_{t+1}
$$

and substituting (5) we finally have:

$$
X^{*}{ }_{t+1}=\alpha+\mu X_{t}+\gamma \varepsilon_{t+1}
$$

where:

$$
\begin{aligned}
\alpha & =\frac{A\left[2 \ln (A \beta)+\left(a_{2} \gamma\right)^{2}\right]}{2\left(a_{1}+a_{2} A\right)} \\
\mu & =\frac{a_{1}(A-\theta)}{\left(a_{1}+a_{2} A\right)}
\end{aligned}
$$


This stochastic difference equation describes the transition of income from youth to old age. In the following we change our perspective in order to use (7) as the equation describing the income dynamics for the whole dynasty. Because of the non-linear relationship between consumption (or human capital) and the $A$ coefficient stated by (5), both $\alpha$ and $\mu$ bear a nonlinearity w.r.t. $A$. In particular, we are interested in identifying thresholds providing $\alpha<=>0$ and $\mu<\Leftrightarrow 1$. If we look at the deterministic part of (7) in fact, the income dynamics depends on these particular values; as an example if $\alpha>0$ and $\mu<1$ then the income achieves, in the long run, a steady state whatever the initial condition. In order to have $\alpha>0$ the inequality $A>\bar{A}=1 / \beta\left(\exp \left(-\left(a_{2} \gamma\right)^{2} / 2\right)\right) \quad$ must hold, while $A>A^{*}=\left((1+\theta) a_{1}\right) /\left(a_{1}-a_{2}\right)$ is necessary for $\mu>1$. The latter implies $a_{1}>a_{2}$, i.e. individuals are more risk averse during youth. Moreover, in order to assure $X_{t+1}>0$ we need $\alpha>0$, which implies, as we said, $A>\bar{A}$. Nevertheless the latter does not involve $A>A^{*}$, so as two dynamical behaviours are possible: for $A \in\left(\bar{A}, A^{*}\right)$ we have $E\left(d X_{t+1} / d X_{t}\right)<1$ - steady state - while for $A>\operatorname{Max}\left(\bar{A}, A^{*}\right) \Rightarrow E\left(d X_{t+1} / d X_{t}\right)>1 \quad$ (perpetual growth). When $A<A^{*}$ all dynasties converge to a deterministic average common equilibrium value $X_{\infty}^{*}$ whatever the initial condition. Dynasties starting from $X_{t}<X_{\infty}^{*}$ grow in mean over time at a falling rate; viceversa, for $X_{t}>X_{\infty}^{*}$, income falls steadily until the deterministic equilibrium point. The rationale for such a result can

\footnotetext{
${ }^{5}$ It is worth stressing that the model does not achieve an endogenous growth despite the CRS assumption on the accumulable factor.
} 
be found in the income and substitution effect; as previously noted, when $A$ is sufficiently low, individuals with a medium-low inheritance prefers investments in human capital rather than in consumption; the lower the inherited income the stronger the effect. For the opposite reason, richer people allocate their inherited income mainly in consumption, which in turn implies a low investment in offspring; by the intergenerational transfer this behaviour induces a fall in the average income dynasty over time, although to a decreasing rate. Under this point of view, income differences in the long run are induced only by the random component, as we shall see in the next section. Viceversa, when $A>A^{*}$, the average income grows over time at an endogenous rate $\mu$ for each dynasty. As noted, for a large $A$ individuals prefer consumption to human capital, whatever the inherited income; yet the high value of $A$ in the production function allows a high second period income despite the low investment in human capital. In such a situation, individuals starting from a higher initial income have a comparative advantage with respect to agents starting from a lower initial value; intergenerational transfers amplify over time such an advantage. This fact implies that the distance between rich and poor individuals tends to get worse over time. In this case the role of the random component is not clear; it will be investigated in the next section.

We can rewrite the above conclusions in a shorter way; starting from equation (5), we can obtain the following expression:

$$
C_{t+1}-C_{t}=\lambda\left(X_{t+1}-X_{t}\right)
$$

with $\lambda=\left(a_{2}(A-\theta)\right) /\left(a_{1}+a_{2} A\right)$. By a backward substitution in the r.h.s. (right hand side) we find: 


$$
C_{t+1}-C_{t}=\lambda\left[\mu^{t}\left(X_{t}-X_{0}\right)+\gamma \sum_{i=0}^{t-1} \mu^{i}\left(\varepsilon_{t+1-i}-\varepsilon_{t-i}\right)\right]
$$

The above expression shows that a change in consumption can be seen as the result of two driving forces: the past change in the deterministic component of the income, i.e. the initial condition, and the cumulative effect of the change in the random noise. If $A<A^{*}$ we have $\mu<1$ and the weight of the initial conditions tends to disappear for a large $t$, leaving the current change in consumption to be driven only by the stochastic component (random walk). On the contrary, for $A>A^{*}$ we have $\mu>1$ and in this case the current change in consumption holds memory of the past dynamics. Although a formal analysis of the moments of the income curve is feasible directly by (7), I find more useful analyze the problem with an approximation in continuous time. In this way it is possible to provide a more rigorous mathematical analysis of the statistical law characterizing the income curve as result of an interacting process between the deterministic and the stochastic component. The approach in continuous time requires some mathematical effort but brings us to a richer class of results; the methodology follows the diffusion process approximation, successfully employed in biology and genetics. The analysis is performed in the following section.

\section{4 - Income Distribution Dynamics}

In this section we are going to take into account all dynasties living in the economy by the introduction of a distribution for the inherited income $X_{t}$. By so doing, we can interpret (7) as the transition equation of the income distribution through two instants. In this way, $X_{t+1}$ comes from a 
convolution between the distribution of $X$ at time $t$ and the normal distribution of the aggregate shock $\varepsilon_{t+1}$. Knowledge of the density function for $X_{t+1}$ provides statistical information about the income distribution. According to this point of view, we can use (7) to study the evolution of the $X_{t}$ distribution modulated by the random component. At this end, we can rearrange (7) as a stochastic finite-difference equation, since $X_{t+1}=\Delta X+X_{t}$ and $\Delta t=1$, i.e. $\Delta X=\left(\alpha+\delta X_{t}+\gamma \varepsilon_{t+1}\right) \Delta t$, where $\alpha=\frac{A\left[2 \ln (A \beta)+\left(a_{2} \gamma\right)^{2}\right]}{2\left(a_{1}+a_{2} A\right)}$ and $\delta=\frac{a_{1}(A-\theta)}{a_{1}+a_{2} A}$, with $\alpha>0$. For $\Delta X, \Delta t \downarrow 0$ we obtain the following ITO linear differential equation ${ }^{6}$.

$$
d X(t)=(\alpha+\delta X(t)) d t+\gamma d W(t)
$$

where $W(t)$ is a standard Wiener process built on a certain probability space $(\Omega, \mathrm{Ft}, \mathrm{P}), \alpha+\delta X$ is the local drift coefficient and $\gamma^{2}$ the local diffusion coefficient. As we said above, the $A$ coefficient is crucial for the dynamics, since it affects the $\delta$ sign. For $A>A^{*}$ we have $\delta>0$ and $\delta<0$ otherwise. In (8), there is a non-zero probability for poor agents to be expelled from the economy ( $X \tau \leq 0$, for some exit time $\tau<\infty$ ) because of uncertainty in the labour market. To avoid such possibility we have the following:

Proposition 1. In order to make the exit time $\tau$ approaching infinity is sufficient a low value for $\gamma$ relatively to $\alpha$ and $\delta$.

${ }^{6}$ Generally speaking, when we approximate a stochastic difference equation by a differential one, the drift coefficient is biased by an extra term which represents the difference between the ITO and the Stratonovich stochastic integration; to avoid this problem a change of probability measure, via Girsanov theorem, is necessary. Nevertheless, 
Proof. See Appendix A1.

Now we are ready to analyze the dynamics of the income distribution by the following

Proposition 2. From (8) the density function for Xt is obtained by the application of a parabolic differential operator (Fokker - Planck equation).

Proof. See appendix A2.

In general such operator is the following:

$$
\frac{1}{2} \frac{\partial^{2}}{\partial x^{2}}[\sigma(x, t) f(x, t)]-\frac{\partial}{\partial x}[\mu(x, t) f(x, t)]=\frac{\partial f(x, t)}{\partial t}
$$

where $\sigma(x, t)$ is the diffusion coefficient of the ITO equation, $\mu(x, t)$ the drift coefficient and $f(x, t)$ the transition density function, i.e. our unknown. Applying (9) to (8), the density function for the income solves the following Fokker - Planck equation:

$$
\frac{\gamma^{2}}{2} \frac{\partial^{2}}{\partial x^{2}} f(x, t)-(\alpha+\delta x) \frac{\partial}{\partial x} f(x, t)-\delta f(x, t)=\frac{\partial f(x, t)}{\partial t}
$$

where $X(t)=x$. Usually, this kind of equations can be solved by an integral transform, as the Laplace or the Fourier transform; in this case, however, both transforms are equivalent since the $x$ variable is defined on the positive semi-axis only (we assume $f(x, t)=0 \forall x<0$ ). Moreover, the same is true for the moments generating function (m.g.f.) $\phi(s, t)=\int_{-\infty}^{+\infty} e^{s u} f(u, t) d u$ which, in this case, corresponds to the Laplace transform, where $s$ is a variable defined in the complex domain.

when the diffusion coefficient does not depend on the process $X(t)$, as in our case, this problem does not raise. 
We use the m.g.f. to reduce the order of the Fokker Planck. Applying the m.g.f. transform to both sides of (10) and integrating, we obtain the following linear, first order partial differential equation:

$$
\left(\frac{\gamma^{2}}{2} s+\alpha\right) s \phi(s, t)=\frac{\partial \phi(s, t)}{\partial t}-\delta s \frac{\partial \phi(s, t)}{\partial s}
$$

which can be integrated by the characteristics method. The auxiliary equations are:

$$
\frac{d t}{1}=-\frac{d s}{\delta s}=\frac{d \phi}{\left(\frac{\gamma^{2}}{2} s+\alpha\right) s \phi}
$$

Two independent solutions are $a=s e^{\delta t}$ and $b=\phi \exp \left(-\left(\frac{\gamma^{2} s^{2}}{4 \delta}+\frac{\alpha}{\delta} s\right)\right)$. The general solution is thus:

$$
\phi(s, t)=\exp \left(\frac{\gamma^{2} s^{2}}{4 \delta}+\frac{\alpha}{\delta} s\right) g\left(s e^{\delta t}\right)
$$

where $g()$ is an arbitrary function which can be eliminated by assigning an initial condition. Equation (12) represents the m.g.f. of the unknown density function $f(x, t)$. We have to note that the exponential function, on the r.h.s., represents the m.g.f. of a normal density with mean $\alpha / \delta$ and variance $\gamma^{2} / 2 \delta$. However, we can know the exact m.g.f. assigning an initial condition $\phi(s, 0)$; we perform this in the next section. 


\section{1 - Two relevant cases}

In the following, we are going to use two different initial conditions for the income distribution in order to rule out the arbitrary function $g()$; in the first one, we assume a normal density function while an exponential law is assumed in the second one. In this way, we are able to characterize the statistical law underlying the income distribution and its temporal evolution. Let us assume as an initial condition the following m.g.f $\phi(s, 0)=\exp \left(\mu s+(\sigma s)^{2} / 4\right)$, i.e. a normal distribution with mean $\mu$ and variance $\sigma^{2} / 2$. In this case, from (12), we obtain the following cumulant m.g.f. $k(s, t)=\log \phi(s, t)):$

$$
k(s, t)=\left[\frac{\alpha}{\delta}\left(e^{\delta t}-1\right)+\mu e^{\delta t}\right] s+\left[\frac{\gamma^{2}}{2 \delta}\left(e^{2 \delta t}-1\right)+\frac{\sigma^{2}}{2} e^{2 \delta t}\right] \frac{s^{2}}{2}
$$

which represents a normal density with mean $\left[\frac{\alpha}{\delta}\left(e^{\delta t}-1\right)+\mu e^{\delta t}\right]$ and variance $\left[\frac{\gamma^{2}}{2 \delta}\left(e^{2 \delta t}-1\right)+\frac{\sigma^{2}}{2} e^{2 \delta t}\right]$. In other words, starting from an initial distribution characterized by a normal density, this law of distribution is conserved over time with time-varying moments. However the dynamic properties of the moments can dramatically change according to the value of the $\delta$ coefficient; in the $\delta<0$ case, i.e. when $A<A^{*}, f(x, t)$ converges towards a steady state (ergodic) normal distribution with mean $\alpha / \delta$ and variance $\gamma^{2} / 2 \delta$. As we can see from (13), this equilibrium distribution has no memory of the initial condition. As previously noted, in this case the deterministic part of the income equation converges to a common steady state and the equilibrium distribution depends only on the random component. 
Viceversa, in the $\delta>0$ case, we observe a steady increase in the moments; the income distribution evolves continually over time without changing the distribution law. We postpone the economic implications of results until section 5 .

If we instead assume an exponential distribution as initial condition, as approximation of a Pareto's law ${ }^{\text {Z }}$, the result we obtain are deeply different. The m.g.f. (Laplace transform) for an exponential density function, with $b$ coefficient, is $\phi(s, 0)=1 /(1+b s)$. With this initial condition the solution for (12) is:

$$
\phi(s, t)=\frac{1}{1+b e^{\delta t} s} e^{\frac{\gamma^{2}}{2 \delta}\left(e^{2 \delta t}-1\right) \frac{s^{2}}{2}+\frac{\alpha}{\delta}\left(e^{\delta t}-1\right) s}
$$

Equation (14) represents the unknown m.g.f. as the product of two Laplace transforms, i.e. as a convolution between an exponential and a normal distribution. The unknown density $f(x, t)$ is the result of two interacting forces: from one side the memory of the initial condition, represented by the exponential function, and, from the other side, the distribution of the labour market shock, whose coefficients now happen to be endogenous. If $\delta<0$ the weight of initial conditions on the dynamics tends to disappear and $f(x, t)$ converges, once more, towards a normal distribution for the above reasons.

Unfortunately, when $\delta>0$, equation (14) does not belong to a known family of distribution laws; in this case an inverse Laplace transform from

\footnotetext{
${ }^{7}$ Unfortunately we can not use directly a Pareto's law since it does not own a Laplace transform.
} 
$\phi(s, t)$ to $f(x, t)$ is necessary. The following proposition allows us to study the problem by the complex analysis:

Proposition 3 The (14) owns a simple pole.

Proof. See Appendix A3.

By the above proposition the unknown density function $f(x, t)$ can be rewritten as:

$$
f(x, t)=\lim _{s \rightarrow S}(s-S) e^{s x} \phi(s, t)
$$

where $S=-1 /(\exp (\delta t))$ is the simple pole for $\phi(s, t)$. Developing the limit, we finally obtain:

$$
f(x, t)=\frac{1}{b e^{\delta t}} e^{s x+\phi\left(e^{2 \delta t}-1\right) \frac{s^{2}}{2}+\psi\left(e^{\delta t}-1\right) s} \quad s=-1 /\left(b e^{\delta t}\right)
$$

which can be rewritten in the following way:

$$
f(x, t)=h(t) \frac{e^{-\frac{x}{b \exp (\delta t)}}}{b \exp (\delta t)}
$$

that is an exponential distribution with parameter $1 /(b \exp (\delta t))$; for $t=0$ we have the initial condition $\frac{e^{-\frac{x}{b}}}{b}($ being $h(0)=1)$.

Once more, we have to remark that, when $\delta>0$, the memory of the initial distribution is particularly relevant for the dynamic process of the income distribution.

\section{2 - A study on the moments}

So far we have identified the dynamic properties of $f(x, t)$ assuming some explicit functional forms about the initial distribution. In this section 
we use (10) to analyze the $f(x, t)$ moments without specification of initial conditions. Nevertheless this kind of analysis does not allow us to identify a statistical law, as in the previous point (except for a normal distribution), so, in general, both analysis are necessary for a complete investigation.

As said, we can calculate the income distribution moments in a relatively direct manner starting from (10). Knowing that the mean value is defined as:

$$
m=\int_{0}^{\infty} x f(x, t) d x
$$

by differentiating w.r.t. time, we obtain:

$$
\dot{m}=\int x \frac{\partial f(x, t)}{\partial t} d x
$$

Substituting (10) in the above expression and integrating by parts, we finally obtain the following ordinary differential equation for the mean value:

$$
\dot{m}=\alpha+\delta m
$$

Following same methodology, we can obtain the differential equations for higher moments:

$$
\begin{aligned}
& \dot{m}_{2}=2 \delta m_{2}+2 \alpha m+\gamma^{2} \\
& \dot{m}_{3}=3 \delta m_{3}+3 \alpha m_{2}+3 \gamma^{2} m \\
& \dot{m}_{4}=4 \delta m_{4}+4 \alpha m_{3}+6 \gamma^{2} m_{2}
\end{aligned}
$$

By solving the above expression, we find the central moments (variance $V$, skweness $S k$, kurtosis $K u$ ) by the well known relations:

$$
\begin{aligned}
V & =m_{2}-m^{2} \\
S k & =m^{3}-3 m_{2} m+2 m^{3} \\
K u & =m^{4}-4 m_{3} m+6 m_{2} m^{2}-3 m^{4}
\end{aligned}
$$


By doing so we find:

$$
\begin{aligned}
& m(t)=e^{\delta t}\left(\frac{\alpha}{\delta}+m(0)\right)-\frac{\alpha}{\delta} \\
& V(t)=V(0) e^{2 \delta t}+\frac{\gamma^{2}}{2 \delta}\left(e^{2 \delta t}-1\right) \\
& S k(t)=S k(0) e^{3 \delta t} \\
& K u(t)=K u(0) e^{4 \delta t}+\frac{3 \gamma^{2}}{\delta} V(0) e^{4 \delta t}-\frac{3 \gamma^{2}}{\delta} V(0) e^{2 \delta t}+\frac{3 \gamma^{4}}{\delta^{2}}\left(e^{4 \delta t}-\frac{e^{2 \delta t}}{2}+\frac{\delta^{2}}{\gamma^{4}}\right)
\end{aligned}
$$

We retrieve some results previously obtained; if $\delta<0$, whatever the initial condition, the income distribution achieves, for a large $t$ a steady state represented by a normal distribution $(S k=0, K u=3)$ with mean $\alpha / \delta$ and variance $\gamma^{2} / \delta$. The situation dramatically changes if $\delta>0$; in this case we find a steady increase in all moments. There is one case only where we observe a constant value for skewness; this is when the initial distribution is characterized by a symmetric law $(S k(0)=0)$; in such a case this property is conserved over time.

\section{5 - Economic implications of results}

The results obtained in the previous paragraph can be summarized in the following way:

a) for $A>A^{*}$ we observe a steady growth in central moments (17) of the income distribution. In this case the unitary mean wage $A$ is sufficiently high to induce a perpetual growth despite a low investment in human capital. This fact especially supports individuals with a higher inherited 
income. The intergenerational transfer accumulates this behavior amplifying initial differences. From here we have a series of consequences:

1. The income distribution dynamics depends on the initial distribution; as (13), (16) and (17) show, there is no convergence towards an equilibrium distribution which is invariant with respect to initial conditions.

2. The growth of the mean value stands for a steady increase of income for each agent. Nevertheless, the growth of the variance implies an increasing dispersion; agents born at time $t$ are richer than the ones previously born, but they are more distant from the current rich agents. Further, asymmetric distribution tends to get worse over time.

3. Under an assumption of homogenous agents we can interpret (8) as the aggregate income equation; the mean growth rate of the economy is endogenously determined by the average human capital remuneration $A$, individual preferences $a_{1}, a_{2}$, and discount rate $\beta$.

b) If $A<A^{*}$, the income distribution converges towards an invariant probability measure whatever the initial conditions; as (17), (13), (14) show, this steady state distribution is characterized by a normal law with mean $\alpha / \delta$ and variance $\gamma^{2} / 2 \delta$. Along the transition path we notice a general reduction in central moments; the economy growth rate, i.e. the mean value of the income distribution, tends to zero and the properties of endogenous growth are ruled out despite the CRS assumption on human capital. Nevertheless the steady state distribution is more equal than the initial one, since, along the transition path, we observe a generalized reduction in variance and 
skewness as well. According to this point of view, the model confirms the presence of a trade-off between growth and equality.

So far we have discussed of results in a dichotomist way, i.e. separating the $\delta>0$ case from the opposite; nevertheless we know that the $\delta$ coefficient is endogenously determined by the parameters characterizing the economy, and in particular by $A$. Assuming invariant individual preferences, any change in the technology, modifying $A$, can affect the income curve dynamics even if, in our scheme, a possible "jump" in $A$ has not an endogenous source ${ }^{8}$. However, several ways to make $A$ endogenous can be imagined. As an example, $A$ could depend on the income dispersion: if the latter grows over time, then a change in $A$ could arise as the result of a social conflict between income classes. If the change in $A$ is such as to induce a negative value in $\delta$, then the income dispersion tends to decline in time, but the mean value also tends to decrease over time; each generation is worse off. This could induce another change in $A$ in order to make $\delta$ positive; the economy starts again towards a steady increase of the moments and so on. The final result is an income distribution alternating falling and increasing patterns in central moments and, in particular, in dispersion, as the empirical observation suggests (see OECD, 1993): the income curve dynamics would be characterized by "episodes" rather than "trends".

So far we have left open the question about the trade-off between growth and equity: we shall fill the gap in the following section.

\footnotetext{
8 In section 6.2 we shall see as $A$ can be endogeneized by means of public expenditure financied by tax revenue.
} 


\section{6 - The redistributive point of view}

In the first part of the paper we have analyzed the economy in a "freemarket" context, stressing the existing trade-off between growth and equality. In this second part we are interested in providing some discussion points about the way such trade-off can be affected by redistributive actions; obviously we are not suggesting a "package" of transfer policies which should be seriously taken into account, but merely to highlight some questions related to social justice. We start with a simple lump-sum policy and subsequently we shall look at a more "traditional" distributive action.

\section{1 - Lump-Sum Policies}

When $\delta>0$, i.e. when income dispersion grows over time, it is possible to reduce inequalities by means of a lump-sum redistribution from top to low classes; such a policy will not affect the growth rate (lump sum policies are not distortionary). First of all, we have to establish a criterion which allows us to separate rich from poor. At this end we can imagine a threshold $\bar{X}$ : each agent whose income is below $\bar{X}$ receive a lump sum subsidy in the first period of life, which is compensated by a lump sum tax on the agents whose income is above $\bar{X}^{9}$. Moreover, since economy is growing over time, we assume that $\bar{X}$ grows at the same average rate $\delta$; finally we will conventionally refer to the low group with $L$ and consequently to the top one as $H$.

\footnotetext{
9 This fact does not imply that the amount of subsidies in the economy is exactly compensated by the amount of taxes, unless Xbar is the median value.
} 
With such a tax-subsidy scheme the individual life cycle is modified in:

$$
\begin{aligned}
& C_{t}+h_{t}=X_{t}+s \\
& X_{t+1}=A h_{t}+\gamma \varepsilon_{t+1}-S \quad \forall X \in\left[\begin{array}{c}
X, \bar{X} \\
-
\end{array}\right]
\end{aligned}
$$

and

$$
\begin{aligned}
& C_{t}+h_{t}=X_{t}-T \\
& X_{t+1}=A h_{t}+\gamma \varepsilon_{t+1}-S \quad \forall X \in(\bar{X}, \infty)
\end{aligned}
$$

where $s$ is the subsidy and $T$ the tax $(s=T)$. By solving the maximization program we obtain the optimal level of $C$ and consequently $X_{t+1}$, viz:

$$
\begin{aligned}
& C^{*}{ }_{t}=\frac{a_{2}(A-\theta)}{a_{1}+a_{2} A} X_{t}-\frac{2 \ln (A \beta)+\left(a_{2} \gamma\right)^{2}-2 a_{2} s}{2\left(a_{1}+a_{2} A\right)} \\
& X_{t+1}=\frac{a_{1}(A-\theta)}{a_{1}+a_{2} A} X_{t}+\frac{A\left(2 \ln (A \beta)+\left(a_{2} \gamma\right)^{2}+2 a_{1} s\right)}{2\left(a_{1}+a_{2} A\right)}+\gamma \varepsilon_{\mathrm{t}+1}
\end{aligned}
$$

where $s>0$ if $X \in[X, \bar{X}]$.

By approximating the above equations in continuos time, we obtain two linear stochastic differential equations, one for each income group:

$$
\begin{array}{ll}
d X=\left(\alpha_{L}+\delta X\right) d t+\gamma d W(t) & X\left(t_{0}\right) \in[X, \bar{X}] \\
d X=\left(\alpha_{H}+\delta X\right) d t+\gamma d W(t) & X\left(t_{0}\right) \in(\bar{X}, \infty)
\end{array}
$$

where: 
$\alpha_{L}=\frac{A\left(2 \ln (A \beta)+\left(a_{2} \gamma\right)^{2}+2 a_{1} s\right)}{2\left(a_{1}+a_{2} A\right)} \quad \alpha_{H}=\frac{A\left(2 \ln (A \beta)+\left(a_{2} \gamma\right)^{2}-2 a_{1} T\right)}{2\left(a_{1}+a_{2} A\right)}$

As it easy to see, the redistributive policy induces only a "scale" effect, making $\alpha_{L}$ higher (hence $\alpha_{H}$ lower) than the corresponding value without subsidy, with $\alpha_{L}>\alpha_{H}$. The redistributive policy does not affect the mean growth rate $\delta$ at all and the final effect is uniquely to make the income classes closer than in the market economy. By considering expectations both in (20) and (21), we obtain two deterministic equations describing the dynamics of the average individuals in the groups, whose solutions are:

$$
m_{i}(t)=e^{\delta t}\left(\alpha_{i}+m_{i 0} \delta\right)-\frac{\alpha_{i}}{\delta} \quad i=L, H
$$

where $m_{i 0}$ is the initial average income in the two groups. By using the above equations it is possible to select the redistributive policy in order to obtain, asymptotically, $m_{L}=m_{H}$. By matching both mean values we find: $\alpha_{L^{-}}$ $\alpha_{H}=\delta\left(m_{H 0}-m_{L 0}\right)$, where $\alpha_{L}>\alpha_{H}$ and $m_{H 0}>m_{L 0}$. Substituting for $\alpha_{L}, \alpha_{H}$, and with $s=T$, we find:

$$
s=T=\frac{\alpha_{L}+\alpha_{H} A}{2 \alpha_{H}} \delta\left(m_{H 0}-m_{L 0}\right)>0
$$

The higher $m_{H 0}-m_{L O}$ (initial inequality), the higher the amount of subsidy (tax) necessary for setting $m_{L}=m_{H}$, i.e. to make as close as possible the classes.

This kind of policy owns interesting contents from a social justice point of view; nevertheless the other side of the coin is represented by the following matter: since the redistributive action does not improve the growth process, why a rich should "pay" for a "poor" agent? It is clear in fact that the policy we are discussing here must be imposed by some central 
authority whose goal is to increase social welfare. It is out of doubt that such a policy, although not Pareto, is Pigou-Dalton improving, because of the concavity of the individual preferences, hence of the social functional: the loss in welfare suffered by well off individuals is less than the gain in less lucky agents. But it is at the same time true that a rich agent never will agree with such a "world view" unless she is particularly open-minded, but this is not required in our market model. Unfortunately we can not close the question by invoking the median voter principle because of the existing conflict between the two income groups. It is trivial to underline in fact, that the necessary democratic support to our distributive policy comes from a pairwise comparison - to tax or not to tax - and, being the majority voting unambiguously the fairest method (K. May, 1952), the result will depend on the median voter behaviour. This latter in turn depends on the effect that the redistributive policy has on her income: if the median voter is herself taxed without being the recipient of the subsidy, then she certainly decides to vote against the redistributive scheme and viceversa. Such a fact brings us back to a question involving a form of central authority which undertakes the decision to include, or not, the median voter among the recipients of the redistributive policy according to some welfare function; in other words we can not hope to solve this social conflict by a democratic mechanism.

\section{2 - Taxes and Public Expenditure.}

In this section we analyze a more traditional policy action which implies majority voting. Let us hypothesize that the coefficient $A$ is not a constant, as assumed so far, but that it depends on public expenditure. In 
other words we assume that the tax revenue affects the individual, and aggregate, technology, providing a scale effect to the human capital. From a slightly different viewpoint, we can say that the public expenditure finances the "quality" of the human capital. Our assumption recalls in part the scheme adopted by Glomm and Ravikumar, (1992), where the tax inflow finances the quality of the educational system. The scheme is the following: at the beginning of the world a young generation is born and each individual is endowed with an initial income according to some distribution function ruled by Nature. Moreover the same Nature decides the initial level of public expenditure in the economy, viz. $A_{0}$. The young generation allocate the endowment between consumption and human capital; in the second period of life the human capital is transformed in income by the usual technology $Y_{l}{ }^{i}=A_{0} h_{0}{ }^{i}$. On this second period income burdens a tax $\tau_{l}$ whose revenue finances the public expenditure, $A_{1}=\tau_{1} \hat{Y}_{1}$, where the hat means the average operator. The after tax income, net of a fixed consumption, provides the bequest for the offspring. The tax rate is chosen by the old generation according to the maximization of the utility function; if they disagree on the tax level then the latter comes from a majority voting mechanism. Under these assumptions the life cycle follows:

$$
\begin{aligned}
& X_{t}^{i}=C_{t}^{i}+h_{t}^{i} \\
& X_{t+1}^{i}=\left(1-\tau_{t+1}\right) Y_{t+1}^{i}-\theta X_{t}^{i} \\
& Y_{t+1}^{i}=A_{t} h_{t}^{i}
\end{aligned}
$$

where $A_{t}=\tau_{t} \hat{Y}_{t}$ is the public expenditure, $\hat{Y}_{t}$ is the average income produced by the old generation at time $t, \hat{Y}_{t}=A_{t-1} H_{t-1}$ and $H_{t-1}$ is the 
average human capital stock. In this way the second period income depends, other than on the individual human capital, also on the public expenditure at time $t$, whose value is correctly observed by each agent in the economy. Since parents take care of offspring in two ways - bequest and public expenditure - we have to modify the individual utility by including the public expenditure level, viz (I omit the superscript $i$ for brevity):

$$
U\left(\tau_{t+1}, X_{t}\right)=U\left(C_{t}\right)+U\left(X_{t+1}\right)+U\left(A_{t+1}\right)
$$

In such a way, each individual expresses her preference about the optimal tax rate $\tau_{t+1}$, given $A_{t}$ and $H_{t}$. The maximization program must be performed in two steps: in the first one, given $\tau_{t+1}$ and $X_{t}$, we obtain the optimal consumption plan and in the second one the optimal tax rate $\tau_{t+1}$ given $A_{t}$ and $H_{t}$. Unfortunately with exponential preferences it is impossible to find an explicit solution to the (23); nevertheless with logaritmic preferences the difficulty is greatly reduced, leaving a reasonable analytical level. In fact, in this case, the optimal tax $r$ ate is the following 10 .

$$
\tau_{t+1}=\frac{3 A_{t}-\sqrt{A_{t}\left(A_{t}+8 \theta\right)}}{4 A_{t}} \in[0,1]
$$

and the corresponding optimal consumption is:

$$
C_{t}^{i^{*}}=\frac{1}{2} \frac{A_{t}+\sqrt{A_{t}\left(A_{t}+8 \theta\right.}-4 \theta}{A_{t}+\sqrt{A_{t}\left(A_{t}+8 \theta\right.}} X_{t}^{i}
$$

where $A_{t}=\tau\left(A_{t-1} H_{t-1}\right)$. The optimal tax rate depends on the whole past taxation profile, hence on the initial conditions, but it depends neither on the

\footnotetext{
${ }^{10}$ Actually the optimal program provides two values of the tax rate but one of them is outside the feasible domain $[0,1]$.
} 
individual income nor on $H_{\mathrm{t}}^{11}$ so that there exists no conflict among individuals: people unanimously agree on the optimal tax rate hence on the level of public expenditure. As $A$ grows over time, the optimal tax rate converges to $1 / 2$, and so does the propensity to consume and accumulate ${ }^{12}$. The public expenditure dynamics follows:

$$
A_{t}=\left(\prod_{i=0}^{t-1} \tau_{t-1} H_{t-(i+1)}\right) A_{0}
$$

which is steadily increasing over time ${ }^{13}$. It must be stressed, anyway, that such a taxation scheme operates an integenerational transfer which does not reduce inequality but does support the growth rate $\frac{14}{14}$ in other words, this kind of taxation scheme is more conceivable for a growth rather than a redistributive policy. If we look at the income growth rate in fact:

$$
X_{t+1}^{i}=\frac{1}{8}\left[\left(A_{t}+\sqrt{\left(A_{t}\left(A_{t}+8 \theta\right)\right)}-4 \theta\right)\right] X_{t}^{i}
$$

it is growing in the public expenditure, which in turn is increasing in the tax rate; moreover it is the same for each agent but, being $A_{t}$ growing over time, this brings to a worsening in inequality.

The dynamical analysis of the whole system is rather complex because of the dependancy of $A$ on the average value $H$ which involve the

\footnotetext{
${ }^{11}$ This is induced by the log-preferences.

${ }^{12}$ It is worth stressing that the propensity to consume depends neither on the individual income nor on the parental income. Under a different point of view, we can say that people have a propensity to consume which is indipendent on the social rank.

${ }^{13}$ Actually the growth property can fail if the initial average income is particularly low respect to the $\theta$; nevertheless such a critical value is too small for being real and in the following we assume that $A$ is growing.

${ }^{14}$ From a slightly different viewpoint, we can look at $A$ as a form of public subsidy to education which improves the "quality" of individuals' human capital; in this case the level of this form of "welfare state" affects positively the growth rate of the economy.
} 
knowledge of the distribution function. Nevertheless, from the (24) it is easy recognize that the system can provide only two alternative dynamics: either convergence to a steady state or a perpetual growth; we are going to show that the latter is the only dynamics compatible with the equations. We proceed by contradiction, i.e. assuming perpetual growth and consequently trying to achieve a contradiction which invalidates our starting assumption. By considering growth in the public expenditure $A$, it is easy to show that the optimal tax rate converges to $1 / 2$ and the following dynamics arises:

$$
\tau^{*}=1 / 2 \quad h_{t}^{i}=1 / 2 X_{t}^{i} \Rightarrow H_{t}=1 / 2 \hat{X}_{t} \quad X_{t+1}^{i}=\alpha A_{t} X_{t}^{i} \Rightarrow \hat{X}_{t+1}=\alpha A_{t} \hat{X}_{t}
$$

where $\alpha$ is a positive constant. By so doing we obtain a simple system of two non-linear first-order difference equations conditional to the hypothesis of perpetual growth:

$$
\begin{aligned}
& A_{t}=\frac{1}{4} A_{t-1} \hat{X}_{t-1} \\
& \hat{X}_{t}=\alpha A_{t-1} \hat{X}_{t-1}
\end{aligned}
$$

We can easily identify a steady state $\left(A^{*}, \hat{X}^{*}\right)$ and linearize the system in the neighbouring of such a point, in order to analyze the long run properties. It is straightforward to show that the spectrum of the Jacobian matrix is composed by two distinct, real, positive eigenvalues lying outside the unit circle, confirming the unstable characteristic of the equilibrium. In other words there is no evidence that the starting assumption of perpetual growth was wrong.

The interesting result of this scheme is that it is able to induce a perpetual growth in any case; differently from the market economy there is not any threshold below which the economy converges toward a steady 
state. Nevertheless, as in the market economy, inequalities grow steadily over time and this is another case of trade-off between efficiency and equity.

The conclusion achieved in this section is true under logaritmic preferences but with a more general function the picture is much more complex. If we adopt exponential preferences in fact the optimal tax level depends on individual income and average human capital in a non-linear relation. In this case each agent expresses a different taxation level according to her maximizing program and a majority voting mechanism decides the level for the economy. So a rather complex dynamics arises depending on the income of the median voter, on the average human capital in the economy, on the initial income distribution and on the past tax evolution. This problem has not an explicit solution and in general a numerical scheme could be employed but the strong relationship between "individual" and "environment" variables makes quite hard to understand the results of a numerical simulation. As an example, figure 2 shows the median tax rate in three different simulations. consideration three agents under three different initial conditions: in the first one the initial income of the median agent coincides with the average value of the endowments - we conventionally label such simulation with Symm. In the second one the median is above the average (simulation Right) and the median agent is below the average in the Left simulation.

\footnotetext{
${ }^{15}$ The exercise is based on the following parameters set: $a_{1}=1, a_{2}=a_{3}=0.5, \theta=0.2, A_{0}=4$. The endowments for the three agents are: $(1,3,5)$ in the Symm simulation, $(1,1.5,5)$ in the Left and $(1,4.5,5)$ in the Right one. The exercise was carried out with the Maple V mathematical package.
} 


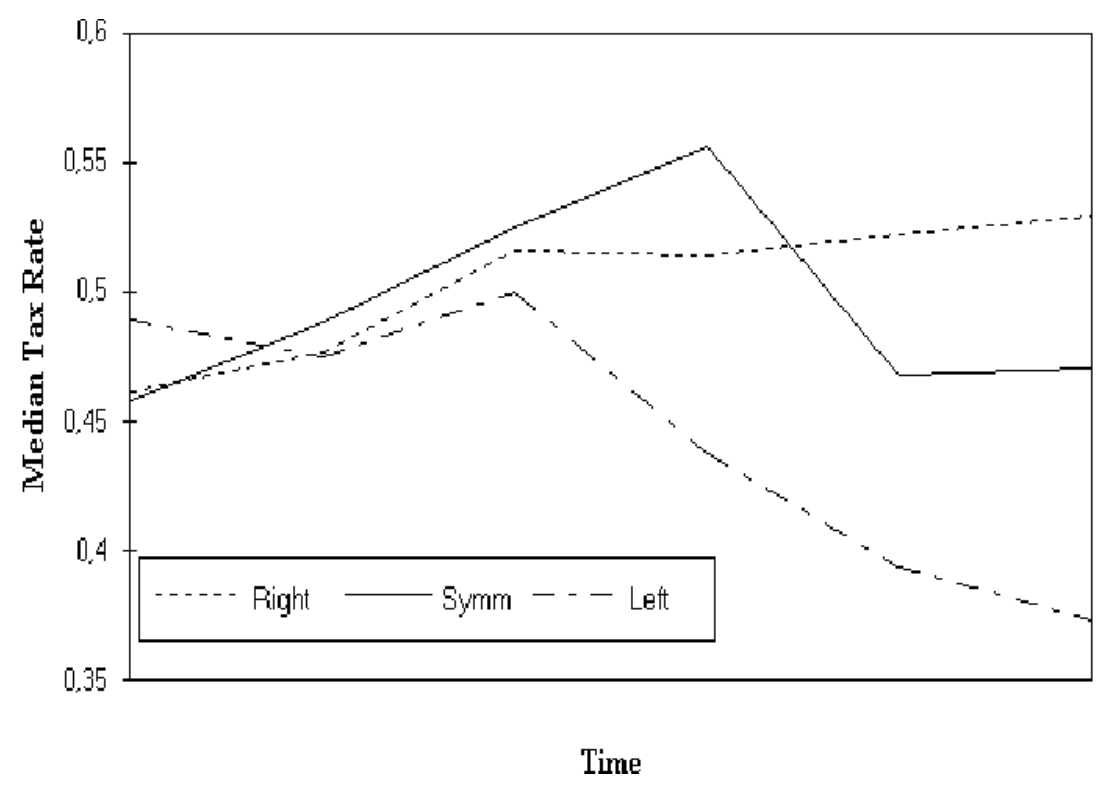

As the figure shows, the tax dynamics is now more complex than the case with logaritmic preferences and strongly depending on the assumption about the median agent. As I said it is not easy to interpret these patterns: however the fastest growth rate is achieved by the Right simulation and this is not a surprise. In the Right simulation the median agent endowment is strongly close to the best endowed individual. In this case the average human capital is high and jointly to a high initial position for two agents out of three allows a relatively high tax level which sustains the growth process. The conclusion is that the richer the economy, the faster the growth process but with a worsening in inequality. The Left simulation is the opposite: two agents out of three are endowed with a low income in order to make low the average. The growth process is still working but at a very low rate: it is interesting however to note the declining behaviour of the tax rate which 
seems to converge to a long run value particularly low - the lowest of the examples. Between these two cases we can insert the Symm simulation which does not seem play any particular role: the growth rate is at an intermediate value between the previous two with a tax level which is not considerably different from the Right simulation; in other words it is not possible to conclude that more equal economies achieve higher growth rate and lower tax rate. However one must be careful in interpreting these simulations; the only clear point is the increasing complexity of the income curve dynamics.

\section{7 - Conclusions}

In this paper we have tried to provide an analytical framework investigating the dynamics of the personal income distribution through investment in human capital. It can be ideally divided in two parts: the first one formalizes a simple market economy and characterizes the income curve dynamics in such a context. The second one has a more political insight, being devoted to discuss redistributive actions. The first part concludes that differentials in wage earnings, induced by differences in accumulated human capital, do matter for the income distribution; intergenerational transfers can amplify inequalities if the economy grows at a positive rate. In such a way, growth and personal income distribution are intimately connected. By varying the assumption about human capital remuneration, it is possible to take into consideration two different dynamics. Under this point of view, the convergence of the income curve towards an ergodic steady state distribution represents only one side of the 
coin; within the same framework, the initial conditions can or can not affect the long run dynamics according to the human capital remuneration. Any change in the labour market, affecting the human capital remuneration, can modify the dynamics of the income distribution; this provides a possible, although partial, answer to the observed changing pattern in the income dispersion. The first part concludes with the presence of a trade-off between growth and equality: in a stylized, maximizing economy, as the one we described, the growth process, in absence of a redistributive policy, involves an increasing inequality. The second part faces the latter point, analyzing the effect of different redistributive policies on growth and inequality. The results can be summarized by the following table:

\begin{tabular}{|l|l|}
\hline Model: & Results: \\
\hline Market (benchmark) & $\begin{array}{l}\text { Growth depending on the threshold A* } \\
\text { Trade-off between growth and equality }\end{array}$ \\
\hline Lump-Sum policies & $\begin{array}{l}\text { Pigou-Dalton improving. } \\
\text { No negative effect on growth rate. }\end{array}$ \\
\hline $\begin{array}{l}\text { Tax on old generation and Expenditure } \\
\text { Public }\end{array}$ & $\begin{array}{l}\text { Steady growth. } \\
\text { A worsening in inequality }\end{array}$ \\
\hline
\end{tabular}




\section{Appendix A1}

In this appendix we prove proposition 1. For an agent starting at $X(0)=x$, the solution of $(8)$ is:

$$
X(t)=\left(x+\frac{\alpha}{\delta}\right) e^{\delta t}-\frac{\alpha}{\delta}+\gamma \int_{0}^{t} e^{\delta(t-s)} d W(s)
$$

So, $X(t)$ is the sum of a deterministic component and a stochastic integral normally distributed, ergo:

$X(t)$ distributed Norm. $\left(\left(x+\frac{\alpha}{\delta}\right) e^{\delta t}-\frac{\alpha}{\delta} ; \frac{\gamma^{2}}{2 \delta}\left(e^{2 \delta t}-1\right)\right) \quad \delta>0$

$X(t)$ distributed Norm. $\left(\left(x-\frac{\alpha}{|\delta|}\right) e^{-|\delta| t}+\frac{\alpha}{|\delta|} ; \frac{\gamma^{2}}{2|\delta|}\left(1-e^{-2|\delta| t}\right)\right) \quad \delta<0$

The application of Chebyshev inequality states:

$$
P\left\{\left|\gamma \int_{0}^{t} e^{\delta(t-s)} d W(s)\right|>\left(x+\frac{\alpha}{\delta}\right) e^{\delta t}-\frac{\alpha}{\delta}\right\} \leq \frac{\gamma^{2} \int_{0}^{t} e^{2 \delta(t-s)} d s}{\left[\left(x+\frac{\alpha}{\delta}\right) e^{\delta t}-\frac{\alpha}{\delta}\right]^{2}}=\frac{\gamma^{2}\left(e^{2 \delta t}-1\right)}{2 \delta\left[\left(x+\frac{\alpha}{\delta}\right) e^{\delta t}-\frac{\alpha}{\delta}\right]^{2}} \quad \delta \in \Re
$$

By making the r.h.s. of the above inequality as small as possible we can reduce the instantaneous exit probability to a negligible value; this is true if $\gamma$ is sufficiently small relatively to $\alpha / \delta$.

A more direct way to the matter consists of using the normality property stated by (A1.1). For a normal variable, it easy to prove that the probability to assume a value outside the $m \pm 3 s$ range, where $m$ is the mean and $s$ the standard deviation, is less than $1 \%$. So, in order to make the instantaneous exit probability less than $1 \%$, we have to satisfy the following:

$$
\left(x+\frac{\alpha}{\delta}\right) e^{\delta t}>3 \frac{\gamma \sqrt{\left(e^{2 \delta t}-1\right)}}{\sqrt{2 \delta}}+\frac{\alpha}{\delta} \quad \delta>0
$$




$$
\left(x-\frac{\alpha}{|\delta|}\right) e^{-|\delta| t}+\frac{\alpha}{|\delta|}>3 \frac{\gamma \sqrt{\left(1-e^{-2|\delta| t}\right)}}{\sqrt{2|\delta|}} \quad \delta<0
$$

which, for large $t$, simplifies to $\left(x+\frac{\alpha}{\delta}\right)>\frac{3 \gamma}{\sqrt{2 \delta}}$ for $\delta>0$, and $\left(x-\frac{\alpha}{|\delta|}\right)>\frac{3 \gamma}{\sqrt{2|\delta|}}$ for $\delta<0$. In general, the latter are easily satisfied if $\gamma$ is small relatively to $\alpha / \delta$.

Q.E.D.

\section{Appendix A2}

This appendix shows how the Fokker Planck equation is obtained. When we are interested in studying the evolution of a probabilistic law, as we are in this case, we must define suitable operators to deal with Markov processes. The evolution of a stochastic process can be viewed in a probabilistic way, as the transition from a state $x$ to another $y$ in a given interval time $t-s, t>s$. In other words, we can think of the stochastic process as a series of "random jump" from a position $x$ at the time $s$, to another state $y$ at the time $t(t>s)$; obviously we can assign a probability to this event, i.e. the transition probability from $(x, s)$ to $(y, t)$. We are interested in the evolution of this probability. When we have to handle stochastic processes assuming values on a continuum, i.e. whose state space is the real-number space, we can not identify the probability of a transition towards a given

\footnotetext{
${ }^{16}$ A more sophisticated approach consists of solving the ordinary differential equation $1=\gamma^{2} / 2 T^{\prime \prime}+(\alpha+\delta x) T^{\prime}, T(0)=0$ (see Ewens, 1979, page 120), where $T$ is the average exit time
} 
value, since, as it is well known, the probability that a continuous random variable assumes a given value $y$ is zero. In this case then, we ask for the probability that the process jumps from $(x, s)$ to $(B, t)$ where $B$ is a borelian on $\Re$. We define $P(s, x, t, B)=P(x(t) \in B \mid x(s)=x)$ as the probability that the process will assume, at the time $t>_{S}$, a state in the $B$ space conditional to the present state $(x, s)$; this transition probability represents a probabilistic temporal evolution law.

For each bounded measurable function $g(x)$ we can define a Markov transition operator $T_{t}$, applied to $\mathrm{g}(\mathrm{x})$, in such a way:

$$
T_{t} g(x)=E_{x} g(X)=\int_{\Re} g(y) P(s, x, t d y)
$$

This operator is the mean value of $g\left(X_{s+t}\right)$ under the condition $X(s)=x$. Roughly speaking, this operator provides the average transition value for the random function $g(X)$. We use this transition operator in order to calculate the derivative of $g(x)$ with respect to time, which is known as the "infinitesimal generator" $A$ for a Markov process:

$$
\operatorname{Ag}(x)=\lim _{t \rightarrow 0} \frac{T_{t} g(x)-g(x)}{t}
$$

The infinitesimal generator provides the mean infinitesimal rate of change of $g(X)$ when $X(s)=x$. Using the transition distribution we can rewrite the infinitesimal generator as follows:

$$
\operatorname{Ag}(s, x)=\lim _{t \rightarrow 0} \frac{1}{t} \int_{\Re}(g(s+t, y)-g(s, x)) P(s, x, t d y
$$

of $X(t)$ from the positive region, and to look for a parametric set which makes $T$ approaching infinity. Yet, this equation can be solved numerically only. 
For a diffusion process $X_{t}$, the transition probability satisfies the following expressions:

$$
\begin{aligned}
& \lim _{t \downarrow_{s}} \frac{1}{t-s} \int_{|y-x| \leq \varepsilon}(y-x) P(s, x, t, d y)=f(s, x) \\
& \lim _{t \downarrow_{s}} \frac{1}{t-s} \int_{|y-x| \leq \varepsilon}(y-x)^{2} P(s, x, t, d y)=\sigma^{2}(s, x)
\end{aligned}
$$

where $f$ is the "instantaneous drift coefficient", which represents the deterministic mean value of the displacement $d X(t)$, and $\sigma^{2}$ is the instantaneous variance (diffusion coefficient).

Expanding (A1.1) by a Taylor series, once w.r.t. $t$ and twice w.r.t. $x$, and using the drift and diffusion coefficients, we finally obtain the Kolmogorov's forward equation, also called Fokker-Planck:

$$
\frac{\partial P}{\partial t}=-\frac{\partial}{\partial y}(f(t, y) P)+\frac{1}{2} \frac{\partial^{2}}{\partial y^{2}}\left(\sigma^{2}(t, y) P\right)
$$

where $P$ stands for $P(s, x, t, d y)$. The solution of this second-order, parabolic partial differential equation provides the temporal evolution for the unknown transition distribution.

\section{Appendix A3}

In this appendix we show that an inverse Laplace transform can be written as equation (15) by complex analysis.

For a function $f(s)=g(s) /(s-a)^{n}$, where $g(s) \neq 0$ and $g(s)$ is analytic on a given region containing $a$, we say that $f(s)$ has a $n$-order pole in $a$; if $n=1$ then we speak about it as a simple pole. If $f(s)$ exhibits a $n$-order pole in $s=a$ but it is analytic overall the other points belonging to a closed ball with 
center in $A$ and contour $C$, then $(s-a)^{n} f(s)$ is analytic on this domain and it owns a Taylor expansion around $a$ :

$$
f(s)=\frac{a_{-n}}{(s-a)^{n}}+\frac{a_{-n+1}}{(s-a)^{n-1}}+\ldots .+\frac{a_{-1}}{(s-a)}+a_{0}+a_{1}(s-a)+a_{2}(s-a)^{2}+\ldots .
$$

This particular series is the Laurent expansion for $f(s)$. The part characterized by negative exponents of $(s-a)$ is the principal part. The Laurent series can be used to investigate the characteristic of the poles. When the principal part shows a finite number of terms and $a_{-n} \neq 0$ while $a_{-n-}$ 1, $a_{-n-2}, a_{-n-3} \ldots .$. are all zero, then $s=a$ is a pole of order $n$.

The $a_{-1}$ coefficient, which is called the residue of $f(s)$ in the pole $s=a$, has a particular importance in the Laurent series. In general, using a Taylor expansion, this particular coefficient is:

$$
a_{-1}=\lim _{s \rightarrow a} \frac{1}{(n-1) !} \frac{d^{n-1}}{d s^{n-1}}\left\{(s-a)^{n} f(s)\right\}
$$

which for a simple pole can be rewritten in an easier way: $a_{-1}=\lim _{s \rightarrow a}(s-a) f(s)$. If $f(s)$ has a simple pole, then integrating the (A3.1) on the $C$ contour we obtain:

$$
\oint_{C} f(s) d s=2 \pi i a_{-1}
$$

where $i$ is the imaginary unit. In other words, the $f(s)$ integration on a closed contour surrounding the simple pole $a$ is equal to $2 \pi i$ times the residual. In general if $\mathrm{f}(\mathrm{s})$ is analytic into and on the contour $C$ of a region $\mathfrak{R}$, apart for a finite number of poles $a, b, c \ldots$ inside $\mathfrak{R}$, then we have: 


$$
\oint_{C} f(s) d s=2 \pi i\left(a_{-1}+b_{-1}+c_{-1}+\ldots\right)
$$

i.e. the integral of $f(s)$ on a contour $C$ is $2 \pi i$ times the sum of the residuals. We are going to use this powerful theorem to demonstrate the inverse Laplace transform.

If we indicate with $f(s)$ the transformed function, then the problem is to look for the not-transformed function $F(x)$. This latter is given by:

$$
F(x)=\frac{1}{2 \pi i} \int_{\gamma-i \infty}^{\gamma+i \infty} e^{s x} f(s) d s \quad x>0
$$

The integration must be performed along a line $s=\gamma$ on the complex plane, so as to stay on the right of each pole; this condition guarantees the existence of the integration.

From the Laplace analysis we know that the (A3.2) can be solved by an integration on the "Bromwich contour" which is a part of the $C$ contour. We have then:

$$
F(x)=\frac{1}{2 \pi i} \oint_{C} e^{s x} f(s) d s
$$

but for the residuals theorem, $F(x)$ is the sum of the $e^{s x} f(s)$ residues for the $f(s)$ poles. Obviously this method is greatly simplified if we have a simple pole for $f(s)$; in this case we have:

$$
F(x)=\lim _{s \rightarrow S}(s-S) e^{s x} f(s)
$$

where $S$ is the simple pole of $f(s)$; this is exactly the (15).

It is worth investigating the type of pole we obtained in (14). This is clearly a simple pole: by the Laurent series we indeed find: 
$\Pi_{1}\left(s+\frac{1}{b \exp (\delta t)}\right)^{-1}+\Pi_{2}+\Pi_{3}\left(s+\frac{1}{b \exp (\delta t)}\right)+\Pi_{4}\left(s+\frac{1}{b \exp (\delta t)}\right)^{2}+\ldots$

where $\Pi_{i}$ are coefficients adequately computed. As it easy to see, the pole $1 /($ bexp $\delta t))$ has order one, i.e. a simple pole.

Q.E.D.

\section{REFERENCES}

Acemoglu, D. 1997. "Matching, Heterogeneity and the Evolution of Income Distribution," Journal of Economic Growth, 2, pp. 61-92.

Alesina, A. and D. Rodrick. 1993. "Distribution, Political Conflict and Economic Growth: A simple Theory and Some Empirical Evidence," in Political Economy, Growth and Business Cycles. Cukierman, Hercowitz and Leiderman, Eds. Cambridge: MIT Press.

Alesina, A. and D. Rodrick. 1994. "Redistributive Politics and Economic Growth," Quarterly Journal of Economics, 109, pp. 465-490.

Arrow, K. 1962. "The Economic Implications of Learning by Doing", Review of Economic Studies, 29, pp. 155-173.

Atkinson, A. B. 1974. The Personal Distribution of Income. London: G. Allen.

Atkinson, A.B. 1995. "Is the Welfare State necessarily an obstacle to Economic Growth?," European Economic Review, 39, pp. 723-730.

Atkinson, A.B. 1996. "Bringing Income Distribution in From the Cold", Presidential Address to the Royal Economic Society, Swansea, UK. 
Benabou, R. 1996. "Inequality and Growth," working paper NBER Nr. 5658 .

Benabou, R. 1996. "Heterogeneity Stratification and Growth, Macroeconomic Implications of Community Structure and School Finance," American Economic Review, 86, pp. 584-609.

Black, D. 1958. The Theory of Committees and Elections. Cambridge: Cambridge University Press.

Cox, D.R. and H.D. Miller. 1965. The Theory of Stochastic Processes. London: Chapman and Hall.

Ewens, W.J. 1979. Mathematical Population Genetics, Berlin: Springer Verlag.

Galor, O. and J. Zeira. 1993. "Income Distribution and Macroeconomics," Review of Economic Studies, 60, pp. 35-52.

Galor, O. and D. Tsiddon. 1997. "The Distribution of Human Capital and Economic Growth", Journal of Economic Growth, 2, pp. 93-124.

Glomm, G. and B. Ravikumar. 1992. "Public versus Private Investment in Human Capital: Endogenous Growth and Income Inequality," Journal of Political Economy, 100, pp. 818-834.

Loury, G.C. 1981. "Intergenerational Transerfs and the Distribution of Earnings", Econometrica, 49, pp. 843-867.

Lucas, R. 1988. "On the Mechanics of Economic Development," Journal of Monetary Economics, 22, pp. 3-42.

May, K. 1952. "A set of Independent Necessary and Sufficient Conditions for Simple Majority Decision", Econometrica, 20, pp. 680-4. 
OECD, 1993. Employment Outlook. Paris: OECD.

Perotti, R. 1993. "Political Equilibrium Income Distribution and Growth," Review of Economic Studies, 60, pp. 755-776.

Perotti, R. 1996. "Growth, Income Distribution and Democracy: What the Data Say," Journal of Economic Growth, 1, pp. 149-187.

Persson, T. and G. Tabellini. 1993. "Growth, Distribution and Politics", in Political Economy, Growth and Business Cycles. Cukierman, Hercowitz and Leiderman, Eds. Cambridge: MIT Press.

Romer, P. 1986. "Increasing Returns and Long Run Growth," Journal of Political Economy, 94, pp. 1002-1037.

Uzawa, H. 1965. "Optimum Technical Change in an Aggregative Model of Economic Growth," International Economic Review, 6, pp. 18-31.

Vaughan, R. 1979. "Class Behaviour and the Distribution of Wealth," Review of Economic Studies, 46, pp. 447-465. 